\title{
简单细胞方位选择性感受野组织形成的 神经网络模型*
}

\section{杨 谦 齐翔林 汪云九 ${ }^{* *}$}

(中国科学院生物物理研究所视觉信息加工研究开放实验室, 北京 100101)

\begin{abstract}
摘要 为了阐明视皮层简单细胞方位选择性感受野形成的动态组织过程, 试图构建 一个由侧膝体神经元和视皮层简单细胞组成的, 且遵从 Hebbian 学习规则的神经网络 模型. 通过该模型来考察简单细胞对自然图像刺激特征的编码过程和神经表达. 结果 表明, 感受野的结构正反映了简单细胞的最优方位选择性, 它也是由非监督学习过程 决定并自组织涌现的. 这还说明简单细胞的方位选择性是在层间细胞的相互作用基础 上动态自组织的结果.
\end{abstract}

\section{关键词 方位选择性 感受野 动态自组织 非监督学习 神经编码}

哺乳动物视觉皮层中的简单细胞代表了视觉皮层信息处理的第一阶段, 它的时空感受野 表达是其对刺激的空间和时间特性选择性地产生发放的结果. 50 年代末以来, Hubel 等人 ${ }^{[1,2]}$ 首 先开创了对视皮层细胞的研究，他们在视皮层 17 区(V1 区)第 4 层上发现了对特殊朝向的条 形光刺激有强烈反应的感受野构型. 但是关于对视觉刺激的方位具有如此强烈的选择性的神 经机制问题, 研究结果却始终是矛盾的, 由此也就产生了两大学派的不同观点 ${ }^{[3]}$ : 其一是 “直 接兴奋性输入会聚说”，认为感受野重叠并排列成一行的外侧膝状体(LGN)细胞的兴奋性输 入，会聚地投射到同一个视皮层细胞，从而使得视皮层细胞对平行于 LGN 细胞感受野所排列 方位的视觉刺激特别敏感 ${ }^{[4]}$; 其二为 “视皮层内相互作用说”，强调视皮层细胞方位选择性主 要取决于视皮层内细胞间的相互作用(正交方位抑制), 它假设外膝体神经元输入对方位选择 性的贡献是非常小的. 这两种假说各自都得到许多的实验结果支持. 近年来, Vidyasagar 等人 ${ }^{[5]}$ 综合以上两种假说, 提出了视皮层简单细胞方位选择性形成的两阶段模型, 每一阶段都涉及 若干不同的神经机制, 说明从 LGN 到视皮层方位选择性由弱到强的神经机制的多样性和动态性.

从上述假说中可以看出，它们都是从细胞感受野的性质出发预测组织机制，而未考虑感 受野的形成过程及其性质都是细胞功能的体现. 视皮层简单细胞感受野的作用就是对外界图 像的特征进行编码, 它的方位选择性其实就表现出了细胞的一种编码功能 ${ }^{[6]}$. 因此, 我们试图 通过考察简单细胞对图像刺激特征的编码过程, 研究简单细胞对图像的编码功能, 以便认识 它的感受野形成的动态组织过程，从而构建简单细胞感受野自组织的计算模型. 该神经网络

1999-05-27 收稿, 2000-01-31 收修改稿

* 国家自然科学基金资助项目(批准号: 39893340-06, 69835020, 39670186)

** 联系人(E-mail: yjwang@ sun5.ibp.ac.cn) 
模型采用 Hebbian 学习规则作为皮层细胞间的映射方式, 并导致了自组织过程的涌现.

神经电生理实验和局部冷冻皮层的全细胞膜片钳实验已经证实 ${ }^{[4,7]}$, 除了颢叶皮层细胞外, 视皮层简单细胞也是按照稀疏编码的方式对视觉图像进行独立成分特征表达的. 这种稀疏编 码的方式表现了自然界中生物提取、处理信息的最经济原则. 许多研究组对此进行了模型方 面的研究 ${ }^{[8,9]}$, 但是, 他们仅从神经元网络如何实现特征编码的角度考虑, 而忽略了这是细胞 感受野本身自组织形成表达的结果.

因此, 本文首先介绍简单细胞感受野自组织形成的理论模型及其数学表达; 其次, 将以 3 幅不同输入图像作为刺激进行模拟, 并将该模型得到的模拟结果作为第 3 部分; 最后, 对模 拟结果进行了分析, 提出我们的看法并进而讨论了影响它的有关因素.

\section{1 感受野自组织形成的数学模型}

\section{1 模型的构建}

简单细胞的感受野在对自然图景的加工中可以被看作为独立的视觉特征检测器, 这个过 程就导致了它们最终构成了一幅图 像的稀疏、分布式表达. 因此, 我们 设计出简单细胞对图像特征进行编 码表达的模型框图(图 1). 该模型包 括两层, 即外侧膝状体(LGN)和初级 视皮层. 视皮层上的简单细胞和 LGN 上的神经元都是按照二维网格 排列, $(k, l)$ 为皮层细胞的空间坐标变 量, $(i, j)$ 为 $\mathrm{LGN}$ 细胞的空间坐标变 量. 视皮层和 LGN 的空间网格按视 网膜拓扑映射来构建, 它满足在视 皮层和 LGN 上相邻的细胞在视网膜 上的位置也必须相邻这一几何拓扑 关系, 坐标 $(k, l)$ 和 $(i, j)$ 用相同的视网 膜拓扑映射坐标给出.

依据生理实验结果 ${ }^{[10,11]}$, 自然 图像在视皮层中的神经表达可以用

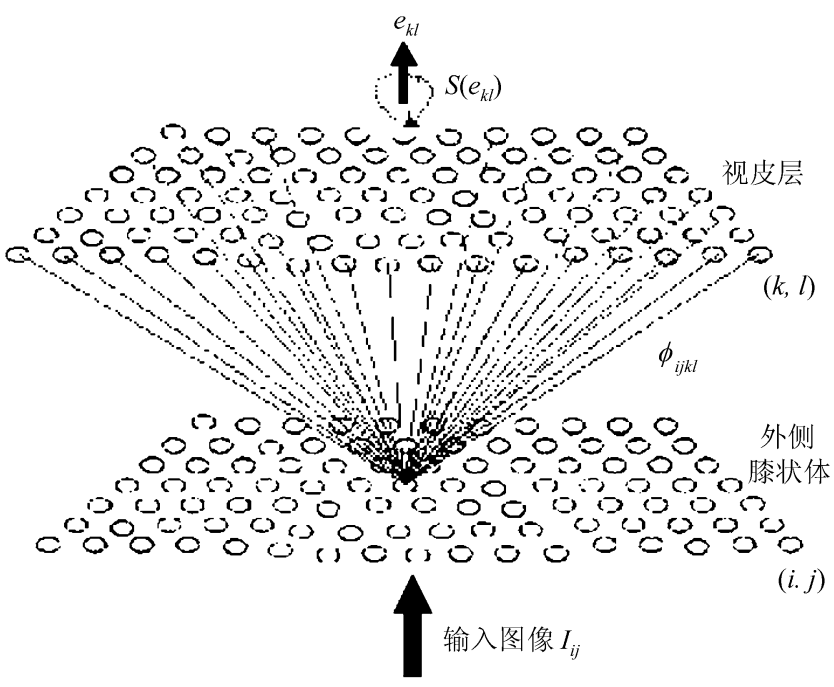

图 1 简单细胞编码的网络模型框图

下层为 LGN 细胞层, 它接受外界图像输入; 上层为简单细胞编码层. 在层中 每一个神经元都有自抑制反馈功能. $\phi_{i j k l}$ 为在整合窗口内层间细胞间 的突触强度的变化

皮层简单细胞的发放 $e_{k l}$ 以它的前馈突触连接强度 $\bar{\phi}$ 为权重的叠加之和来描述. 这里, 简单细 胞不接受来自其他皮层内细胞的输入, 故图像的表达可以写成:

$$
I_{i j}=\sum e_{k l} \phi_{i j k l}+\varepsilon,
$$

$I_{i j}$ 为刺激图像的矩阵表达中的某个像素点上的灰度值, $0 \leqslant k \leqslant M_{1}, 0 \leqslant l \leqslant N_{1} . e_{k l}$ 是简单细胞神经 编码矩阵表达中位于某个空间坐标上的编码值, $0 \leqslant i \leqslant M_{2}, 0 \leqslant j \leqslant N_{2}$. $\phi_{i j k l}$ 是简单细胞和 LGN 细 胞之间的 $\left(M_{1} \times N_{1}\right) \times\left(M_{2} \times N_{2}\right)$ 突触强度连接矩阵, 表达了简单细胞感受野的空间结构. 这里 $\left(M_{1} \times N_{1}\right)$ 是简单细胞的个数, $\left(M_{2} \times N_{2}\right)$ 是 LGN 神经元的个数. 根据生理实验, V1 区第 4 层 简单细胞的数目大大超过外侧膝状体神经节细胞数目, 也就是意味着 V1 区简单细胞的编码 
表达空间的维数大于 LGN 细胞输入表示空间的维数, 这在数学上就是一种超完备表达. $\varepsilon$ 为 外界 Gauss 噪音.

\section{2 模型中基函数的选择}

由生理实验得知 ${ }^{[12]}$, LGN 神经节细胞的感受野呈同心圆型, 即各向同性, 并且附近的细 胞感受野形状相似, 只是位置不同. 因此在模型中采用二维 Gabor 函数作为母函数 ${ }^{[13]}$, 构造 一系列子波表达的同心圆型 LGN细胞的感受野来作为输入层, 用简单细胞作为编码的表达层, 由 LGN 细胞感受野会聚构成皮层简单细胞感受野的初始时空结构, 其二维 Gabor 函数母函数 的表达式为

$$
\begin{gathered}
G(i, j)=d \cdot \cos (2 \text { ð } \omega) \cdot \mathrm{e}^{-\frac{\tilde{a}^{2}}{\sigma^{2}}}, \\
\gamma=\sqrt{(i-c \cdot k)^{2}+(j-c \cdot l)^{2}},
\end{gathered}
$$

LGN 细胞感受野的子波表达为

$$
\begin{aligned}
& \phi_{i j k l}=G\left(x^{\prime}, y^{\prime}\right), \\
& x^{\prime}=\left(i-l_{h}\right) \cos \theta+\left(j-l_{v}\right) \sin \theta, \\
& y^{\prime}=-\left(i-l_{h}\right) \sin \theta+\left(j-l_{v}\right) \cos \theta,
\end{aligned}
$$

这里 $G(i, j)$ 为 2-D Gabor 函数描述, $(i, j)$ 为 LGN 层细胞的空间坐标, $(k, l)$ 是皮层简单细胞空间 坐标, $d$ 为比例常数, $\omega$ 为感受野的空间频率, $\sigma^{2}$ 为感受野空间方差, 表示感受野的大小, $c$ 为超 完备表达的倍数大小, $\gamma$ 为皮层和 LGN 层之间细胞坐标的径向距离, $x^{\prime}, y^{\prime}$ 为子波表达的转换 变量, $l_{h}, l_{v}$ 为空间展开中心的平行移动距离, $\theta$ 为旋转角.

定义网络的能量函数为

$$
\begin{gathered}
E\left(I, e_{k l} \mid \phi_{i j k l}\right)=\left\|r_{i j}\right\|_{L_{2}}^{2}+\lambda \sum_{k, l} S\left(e_{k l}\right), \\
r_{i j}=I_{i j}-\sum_{k, l} e_{k l} \phi_{i j k l},
\end{gathered}
$$

表示自组织调节过程中的残差图像; $\lambda \sum_{k, l} S\left(e_{k l}\right)$ 表示简单细胞的自抑制作用, $\lambda$ 为自抑制作用 强度系数, $S\left(e_{k l}\right)$ 为简单细胞的自抑制函数. 由此得到感受野动态组织的演化方程:

$$
\phi_{i j k l}^{*}=\arg \min _{\phi_{i j k l}}\left\langle\min _{e k l} E\left(I, e_{k l} \mid \phi_{i j k l}\right)\right\rangle .
$$

方程(6)意味着在细胞对刺激图像进行完全编码时, 网络的目标就是找到一个能使能量函 数达到最小的相应的基函数集合 $\phi^{*}$, 故方程(6)可以重写为

$$
\phi_{i j k l}(t+1)=\phi_{i j k l}(t)+\eta\left\langle e_{k l} r_{i j}\right\rangle,
$$

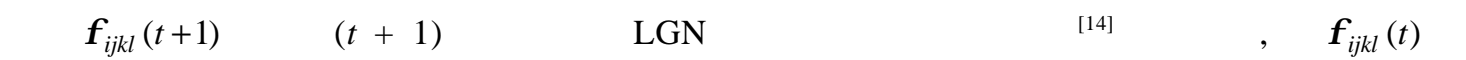
定义了 $t$ 时刻的动态突触的强度值. 由于细胞的动态突触的变化相对于编码表达而言是一个 慢变量, 所以 $\left\langle e_{k l} r_{i j}\right\rangle$ 表示在一个时间窗口中细胞间突触联结强度变化的平均值. $\eta$ 是经典的 Hebbian 学习规则中的学习速率. 


\section{3 模型的网络算法}

我们用图像 $\left\{I_{i j}\right\}$ 作为视觉输入刺激, 由此可以产生简单细胞的初始编码表达的序列

$$
e_{k l}^{i n i t}=\sum_{i, j} \phi_{i j k l} I_{i j} .
$$

依据生理实验，简单细胞的自抑制调谐响应是具有自适应衰减的函数，所以细胞的稀疏 函数取为 $S\left(e_{k l}\right)=-\exp \left(-e_{k l}^{2}\right)$. 为了更能说明自组织涌现的基本机制, 在模拟中依然采用完备 方式来定义编码空间的比例, 即 $M_{1}=M_{2}, N_{1}=N_{2}$. 由于细胞对视觉刺激的编码是以细胞的 发放作为其表达，所以在仿真中刺激图像的像素必须作归一化处理，即

$$
I_{i j}^{\prime}=\frac{I_{i j}}{\max \left\{I_{i j}\right\}} .
$$

稀疏变化系数 $e_{k l}$ 是随输入图像刺激而变化的一个快变量, 故可以得到简单细胞编码的演化方 程为

$$
e_{k l}(t+1)=e_{k l}(t)+\sum_{i, j}\left(\phi_{i j k l} r_{i j}\right)-\lambda S^{\prime}\left(e_{k l}\right) .
$$

在调节过程中, 为了使 $\phi_{i j k l}$ 在突变点处不会发散, 保持细胞突触变化的连续性, 必须给定 约束条件. 因为 $\phi_{i j k l}$ 和 $e_{k l}$ 是相互制约的, 所以取前后两次的 $\phi_{i j k l}$ 模之比动态地随 $e_{k l}$ 在时间窗口 内的平均值而变化来作为归一化约束条件, 即

$$
\begin{gathered}
\phi_{i j k l}^{\mathrm{new}}(t+1)=v \phi_{i j k l}^{\mathrm{new}{ }^{\prime}}(t+1), \\
v=\frac{1}{\mu \sum_{i, j, k, l}\left|\phi_{i j k l}(t)\right|^{2}} \\
\mu=\left[\frac{\left(\sum_{w_{t}} \sum_{k, l} e_{k l}^{2}(t+1)\right) / w_{t}}{\sigma_{I}^{2}}\right]^{\alpha},
\end{gathered}
$$

$\sigma_{I}^{2}$ 为输入刺激图像的方差, $w_{t}$ 为快慢变量整合窗口的大小, $\phi_{i j k l}^{\text {new }}$ 为学习过程中突触的最新 强度, $\phi_{i j k l}^{\text {new }}$ 为它经过归一化后的值, $\phi_{i j k l}^{\text {old }}$ 为突触前一时刻的强度. 这里取 $\alpha=0.01, \lambda$ 为原始 图像方差的 0.1 倍, 学习速率 $\eta$ 为 0.05 .

当细胞在编码刺激特征时，整合窗口 $w_{t}$ 表示层间细胞之间突触强度的变化率，它在生理 上具有明确的意义. 依据生理实验 ${ }^{[15]}$ 结果, 在模型中整合窗口长度定为 20 次迭代步数.

\section{2 计算机模拟结果及分析}

我们自 Lenna 图像中截取 3 幅 $8 \times 8$ 像素的图像, 分别作为输入图像 I, II, III, 其中图 像 I 的朝向信息为 45 度，图像 II 的朝向信息为 90 度，而作为对比使用的子图像 III 没有明显 的朝向信息. 模型中用 64 个单元作为完备表达, 以保证表达维数与输入维数的相同. 在迭代 中当能量评价函数小于 $2 \%$ 时, 认为此时精度已经达到要求, 因此分别得到图像 I, II, III的简 
单细胞编码表达(图 2). 图 2b,2c 和 $2 \mathrm{~d}$ 表明, 在图像 I, II , III 完全重构的基础上, 细胞编码后 得到的细胞发放表达的密集度比初始表达分布大大地减小了. 为了更精确地刻画简单细胞编 码的稀疏程度, 我们得到随时间变化的实时细胞发放数目图和相对应的网络能量变化图, 如图 3 所示. 从图中可以看出, 随着迭代次数的增加, 网络越来越精确地重构原始输入图像. 在完全

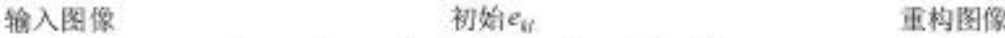

(a)

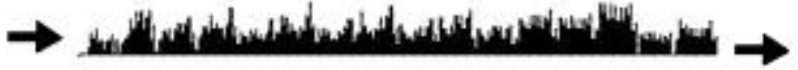

(b)

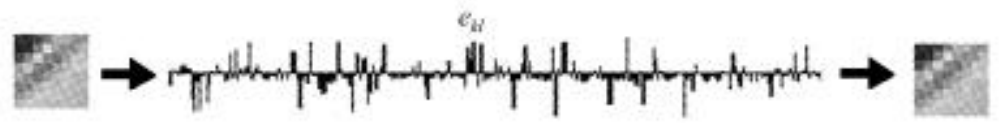

(c)

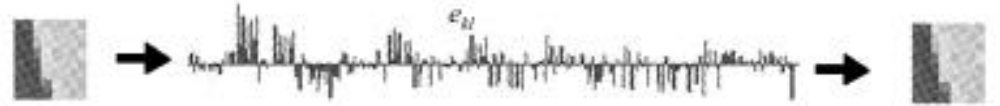

(d)

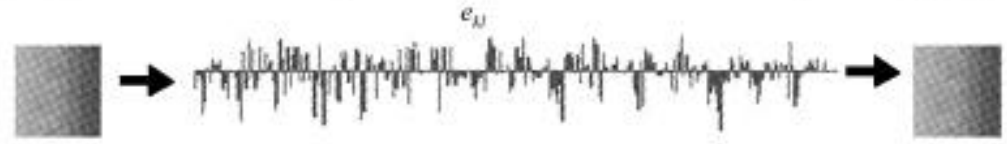

图 2 视皮层简单细胞对不同输入时的编码响应

(a) 简单细胞集群顺序排列的一维编码序列的初始状态. (b), (c)和(d)中各图都包括 3 个部分: 第 1 列为 $8 \times 8$ 像素的输入图像; 第 2 列为当误差小于 $2 \%$ 时简单细胞群顺序排列的一维编码 序列的状态; 第 3 列为此时网络的重构图像
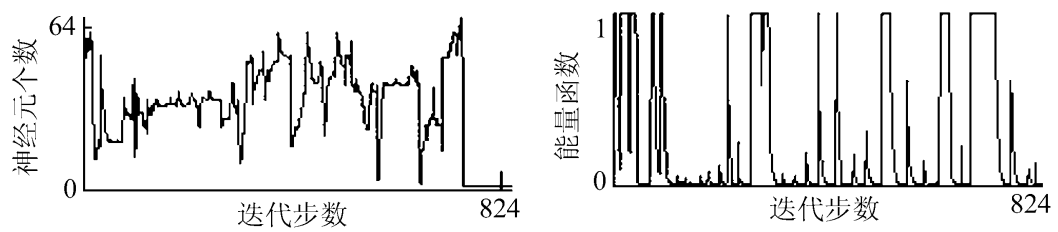

(a)
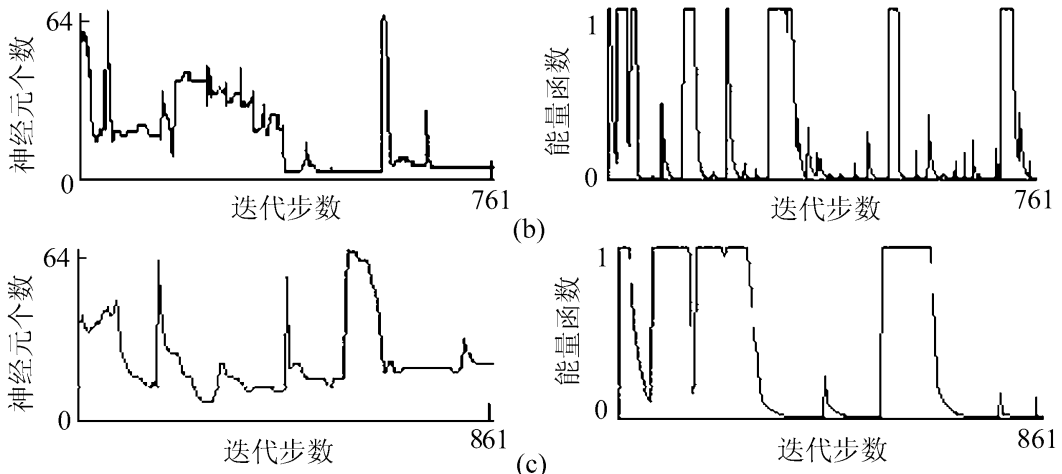

图 3 简单细胞在其感受野自组织形成过程中, 参与编码的细胞数目及其相应 的网络能量函数变化图

(a), (b)和(c)中第 1 列各图为当输入模式分别是图像 I, III, III 时简单细胞参与编码的细胞数目, 而第 2 列各图为相应的网络能量函数的变化. 横坐标为迭代次数, 简单细胞层的细胞总数为 64 
重构图像时, 网络能量趋于最小. 与此同时, 简单细胞群体中产生发放的细胞个数也达到最小, 群体的稀疏度得到提高. 但是不同的输入图像得到的结果不完全一样, 图像的朝向信息越强, 则稀疏表达的稀疏度就越大. 比如, 当输入图像是图像 I 和 II 时, 参与编码的细胞数目就比图 像 III 时的少. 这些结果表明，在输入图像的信息没有改变的基础上，参与简单细胞编码表达的 细胞数的减少体现了简单细胞编码图像主要信息成分的功能. 由于在模型中简单细胞之间并 没有相互作用的关系，所以简单细胞稀疏编码的就是图像信息表达的独立成分.

由此可以得出, 简单细胞在对外界图像编码的过程中, 感受野的空间结构随时间而进行 自组织过程(图 4). 尽管对不同输入图像的编码中, 网络能量达到最小的演化步数不一样, 而 图像 I 和 II 作为输入时，仍表现出从同心圆构型感受野动态组织形成简单细胞朝向检测感受 野构型的过程. 然而作为对比使用的图像 III，由于没有明显的朝向信息，所以感受野并没有自 组织演化出朝向检测构型. 这个对比的模拟实验充分说明了当动物出生后, 其视皮层简单细 胞感受野的方位选择性不仅是由基因控制形成的, 而且还受自然环境中视觉刺激的调制. 也 就是说，刺激图像的朝向信息能使细胞的感受野由具有各向同性感受野的细胞的简单会聚， 经过层间突触的动态组织而最终形成方位选择性.

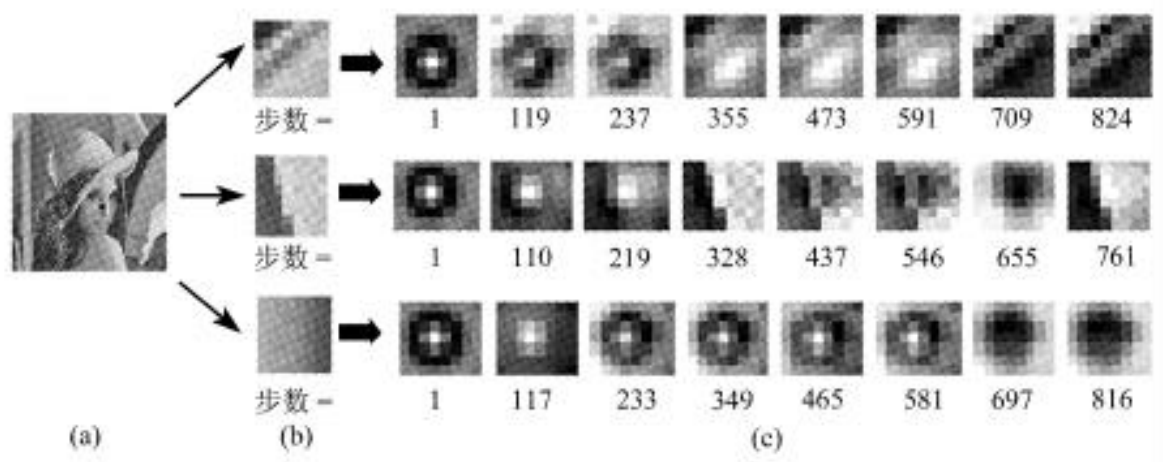

图 4 简单细胞时空感受野形成过程的演化图

(a) 原始Lenna图像; (b) 从原始图像上截取的 3 幅 $8 \times 8$ 图像 I, III，III; (c) 位于简单细胞层中 $(12,26)$

位置上的简单细胞, 分别对图像 I, II, III进行稀疏编码时的感受野自组织过程, 每幅图下面的 数目是此时的迭代步数

但是, 对于同一个视觉刺激, 并不是所有的表达层内的简单细胞都能形成朝向检测. 针对 3 幅不同的图像, 统计得到完全重构图像以后能够形成方位选择性感受野的细胞比例分别为 $83.7 \%, 82.4 \%, 0.4 \%$. 对于同一个图像刺激, 这些简单细胞能够由各向同性的感受野自组织形 成方位选择性感受野以及没有形成的比例分别如图 5 所示, 说明了刺激的朝向信息对形成简 单细胞的方位选择性的重要作用. 这个结果支持了 Hubel 等人在 V1 区既发现了有同心圆构 型的简单细胞感受野，也有条状构型的朝向感受野的实验结论. 同时也说明刺激图像和投射 的自组织过程在很大程度上影响方位选择性感受野的形成.

\section{3 讨论}

视皮层简单细胞在动态学习过程中的编码表达, 说明了简单细胞感受野的方位选择性结 构是由在空间上各向同性感受野结构自组织演化构成的. 该模型的算法在描述朝向映射的模 型和细胞突触分布模式的演化模型之间建立了一种联系, 并使我们得到如下的认识. 首先, 模 
(a)

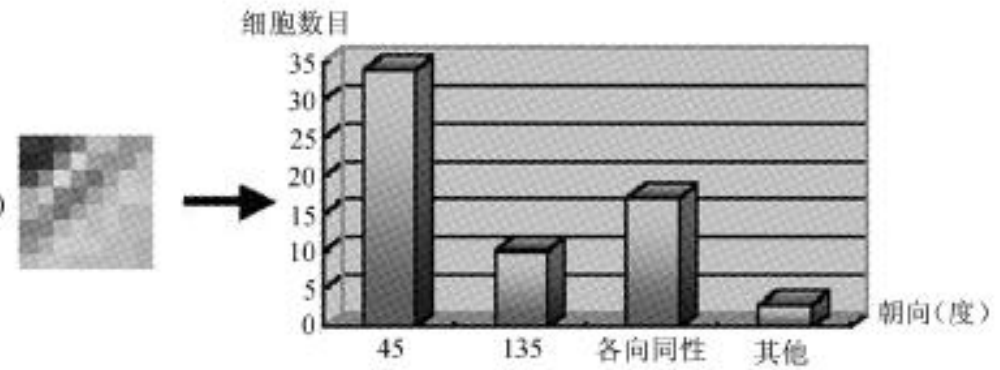

细胞数目

(b)
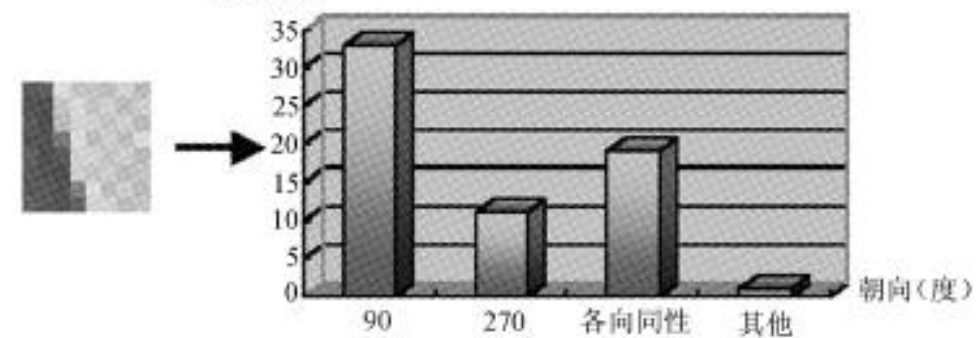

细胞数目

(c)

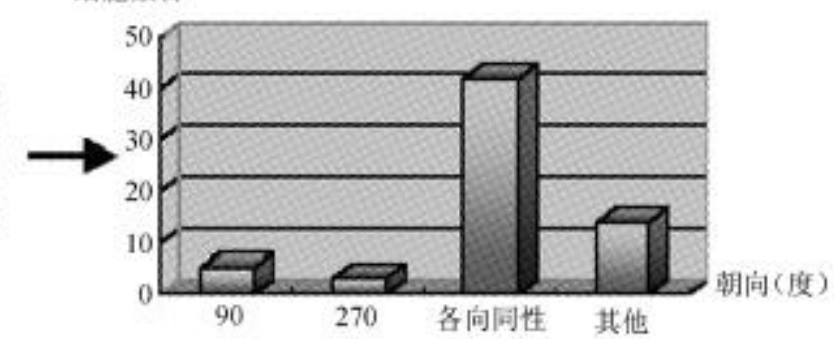

图 5 简单细胞对不同输入图像涌现出方位选择性的比例

左列是不同的输入图像. (a) 对于输入图像 I 涌现 $45^{\circ}, 135^{\circ}$, 各向同性和其他方向的简单细 胞比例. (b) 对于输入图像 II 涌现 $90^{\circ}, 270^{\circ}$, 各向同性和其他方向的简单细胞比例. (c) 对于 输入图像 III 涌现 $90^{\circ}, 270^{\circ}$, 各向同性和其他方向的简单细胞比例. 右图的水平方向是 方位选择性的种类, 垂直方向是形成方位选择性的细胞个数

型的模拟结果表明, 视皮层简单细胞感受野方位选择性的形成是在对视觉环境中刺激图像所 采用的稀疏编码策略和完全表达方式密切相关; 其次, 简单细胞方位选择性的形成还依赖于 视觉刺激图像中刺激边缘的朝向信息. 另外, 皮层内的细胞输入对于简单细胞方位选择性感 受野的形成并不起重要作用. 当然, 并不是所有的简单细胞都能够形成方位选择性, 这可能与 LGN 神经元突触输入的空间分布模式有一定关系.

我们给出了简单细胞方位选择性感受野自组织涌现的分析, 并模拟了简单细胞方位选择 性感受野形成的演化过程. 因此，细胞的位置和快、慢变量之间的整合窗口大小就会影响自 组织过程. 实际上, 整合窗口的大小与动态演化的单位时间、突触前和突触后电位的传递速 度直接相关. 在我们的仿真中, 针对不同的输入图像选取了几种不同的整合窗口大小来进行 模拟, 得到的结果如图 6 所示. 结果表明, 20 个迭代步数左右大小的时间整合窗口能够使更多 的简单细胞形成方位选择性感受野, 这与电生理实验得到的细胞突触信息整合的时间窗口大 小基本一致. 但是它的明确生理意义还有待于更多的电生理实验数据, 特别是有关突触前和 突触后电位传导实验的支持. 


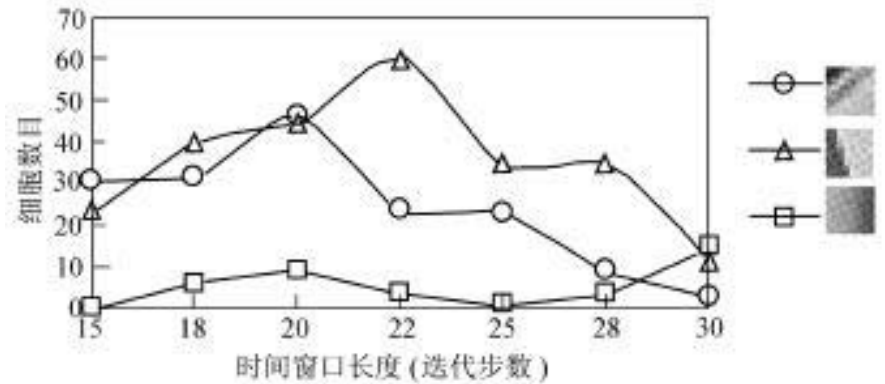

图 6 在不同大小的整合窗口下, 能够形成方位选择性的简单细胞数目统计图 横坐标为时间整合窗口的大小, 单位为迭代步数, 纵坐标为能够形成方位选择性的简单细胞的数目. $\bigcirc, \Delta, \square$ 分别表示在不同输入模式下、整合窗口不同时, 形成方位选择性的简单细胞的个数

另外, 在我们的模拟中并不是所有的简单细胞都能形成方位选择性感受野, 它直接依赖 于 LGN 输入的空间分布. Reid ${ }^{[15]}$ 和 Champman ${ }^{[16]}$ 关于皮层细胞的 LGN 输入的实验强有力地 支持了 Hubel 等人的原始模型的一个主要方面, 即简单细胞感受野的方位选择性由 LGN 的 ON 型和 OFF 型感受野的空间分布调制增强. 这个实验与 Ferster ${ }^{[4]}$ 的实验结果以及用我们提出的 网络模型的模拟结果, 都支持了 Hubel 等人关于仅仅需要 LGN 的空间输入就能增强和调制方 位选择性. 在此, 我们还强调在给出的编码学习算法中不需要皮层内神经元的相互连接, 认为 简单细胞的方位选择性是由于层间细胞的作用决定的, 与层内细胞的相互作用无关. 这一点 与 Chung 等人 ${ }^{[17]}$ 的最新实验结果相吻合, 并有所发展, 主要是当动物出生后, 视觉环境中刺 激的朝向信息通过动态突触映射对简单细胞感受野方位选择性的形成具有很大影响. 而增加 层内局部细胞之间的相互作用是否可以使朝向调谐曲线更加尖锐化, 将是我们下一步数学模 拟实验所要进行的工作.

\section{参考文献}

1 Hubel D H, Wiesel T N. Receptive fields of single neuron in the cat striate cortex. Journal of Physiology, 1959, 148: 574 591

2 Hubel D H, Wiesel T N. Functional architecture macaque monkey visual cortex. Proc Roy Soc B, 1977, 198: 1 59

3 寿天德. 视觉信息处理的脑机制. 上海: 上海科技教育出版社, 1997. 188 197

4 Ferster D, Chung S, Wheat H. Orientation selectivity of thalamic input to simple cells of cat visual cortex. Nature, 1996, 380: 249 252

5 Vidyasagar T R, Pei X, Volgushev M. Multiple mechanisms underlying the orientation selectivity of visual cortical neurons. TINS, 1996, 19: 272 277

6 Artun O B, Shouval H Z, Cooper L N. The effect of dynamic synapses on spatiotemporal receptive fields in visual cortex. Proc Natl Acad Sci USA, 1998, 95: 11 999 12 003

7 Rolls E T, Tovee M J. Sparseness of the neuronal representation of stimuli in the primate temporal visual cortex. J Neurophysiology, 1995, 73: 713 726

8 Olshausen B A, Field D J. Sparse coding with an overcomplete basis set: A strategy employed by V1? Vision Research, 1997, 37: $3311 \sim 3325$

9 Bell A J, Sejnoswski T J. The "Independent components" of natural scenes are edge filters. Vision Research, 1997, 37 : 3 327 3338

10 Dan Y, Atick J J, Reid R C. Efficient coding of natural scenes in the lateral geniculate nucleus: experimental test of a computational theory. Journal of Neuroscience, 1996, 16: 3 351 3 362 
11 Field D J. Relations between the statistics of natural images and the response properties of cortical cells. Journal of the Optical Society of America A, 1987, 4: 2 379 2 394

12 DeAngelis G C, Ohzawa I, Freeman R D. Receptive filed dynamics in the central visual pathway. TINS, 1995,18: 451 458

13 Wang Y J, Qi X L, Chen Y Z. Simulations of receptive fields dynamics. TINS, 1996, 19: 385 386

14 Blais B S, Intrator N, Shouval H, et al. Receptive field formation in natural scence environments: comparison of single-cell learning rules. Neural Computation, 1998, 10: 1 797 1 813

15 Reid R C, Alonso J M. Specificity of monosynaptic connections from thalamic to visual cortex. Nature, 1995, 378: 281 284

16 Champman B, Zahs K R, Stryker M P. Relation of cortical cell orientation selectivity to alignment of receptive fields of the geniculocortical afferents that arborize within a single orientation column in ferret visual cortex. J Neurosci, 1991, 11: $1347 \sim 1358$

17 Chung S, Ferster D. Strength and orientation tuning of the thalamic input to simple cells revealed by electrically evoked cortical suppression. Neuron, 1998, 20: 1 177 1 189 